\title{
The Best Interval Representation of Fuzzy S-Implications and Automorphisms
}

\author{
Benjamín C. Bedregal, Regivan H. N. Santiago, Renata H. S. Reiser, Graçaliz P. Dimuro
}

\begin{abstract}
The aim of this work is to analyze interval fuzzy S-implications and interval automorphisms. Starting from any fuzzy S-implication, it is shown how to obtain an interval fuzzy S-implication canonically. We proved that the such interval fuzzy $S$-implications meet the optimality property and preserve the same properties satisfied by the fuzzy S-implication. In addition, commutative diagrams are used in order to relate fuzzy $S$-implications to interval fuzzy $S$-implications, and to understand how interval automorphisms act on interval $S$ implications, generating other interval fuzzy $S$-implications.
\end{abstract}

\section{INTRODUCTION}

The tolerance for imprecision and the ability to make decisions under uncertainties of control systems provide the seminal motivation for development of fuzzy set theory. Fuzzy logic has been developed as a system of formal deductive systems with a comparative notion of truth to formalize deduction under vagueness. Thus, fuzzy logic gives foundations for approximate reasoning using imprecise propositions based on fuzzy set theory. And, all these matters involve the knowledge of the basic characteristics of approximative reasoning processes response from the viewpoint of uncertainty or incomplete available information and knowledge of the parameters that affect human reasoning and need to be subjected to scientific investigation.

The extension of classical logic connectives to the real unit interval is fundamental for the studies on fuzzy logic and therefore is essential to the development of fuzzy systems. This extension must preserve the behaviors of the connectives at the interval extremes (crisp values) and important properties, such as commutative and associative properties, which result in the notions of triangular norms and triangular conorms. Fuzzy implications play an important role in fuzzy logic, both in the broad sense (heavily applied to fuzzy control, analysis of vagueness in natural language and techniques of soft-computing) and in the narrow sense (developed as a branch of many-valued logic which is able to investigate deep logical questions). However, there is no consensus among researchers which extra properties fuzzy implications should be satisfied. In the literature, several

Benjamín C. Bedregal and Regivan H. N. Santiago are with the Laboratory of Logic and Computational Intelligence, Department of Informatics and Applied Mathematics, Federal University of Rio Grande do Norte, Campus Universitário s/n, Lagoa Nova, Natal-RN, Brazil, CEP 59.072-970 (phone: +55 84 32153814; fax: +55 84 32153813; email: \{bedregal,regivan\}@dimap.ufrn.br).

Renata H. S. Reiser and Graçaliz P. Dimuro are with the Graduate Programme in Computer Science, Informatics School, Catholic University of Pelotas, Rua Feliz da Cunha, 412, Centro, Pelotas-RS, Brazil, CEP 96.010-000 (phone: +55 53 32848227; fax: +55 53 32253105; email: \{reiser, liz\}@ucpel.tche.br). fuzzy implication properties have already been considered and their interrelationship with the other kinds of connectives are generally presented. There exist three main classes of fuzzy implications associated to fuzzy connectives named Rimplications, S-implications and QL-implications which are generated by t-norm, t-conorm joint with fuzzy negation and $\mathrm{t}$-norm together with t-conorm and strong fuzzy negation, respectively.

On the other hand, the correctness and optimality of interval mathematics have been applied in technological [5] and scientific computations [13] to provide accuracy of calculations together with automatic and rigorous control of digital error of numerical algorithms [20]. In this sense, interval computation is adequate to deal with the imprecision of the input values or caused by the round errors which occur during the computation [27], [1]. The interval mathematics is another form of information theory which is related to but independent from fuzzy logic. However, when intervals can be considered a particular type of fuzzy set or when the interval degree of membership is considered to be an imprecision in the belief degree of a specialist, it seems natural and interesting to deal with the interval fuzzy approach.

Among several papers connecting these areas (see, e.g., [28], [10], [26], [14], [24], [18]), we adopted Bedregal and Takahashi work's [6], [7], where interval extensions for the fuzzy connectives, considering both correctness (accuracy) and optimality aspects, were provided [31].

The aim of this present work is to introduce an interval generalization of S-implications, and to show that the action of interval generalizations of automorphisms introduced in [18], [19] preserve the interval generalizations of S-implications. We present how to construct interval S-implications as the best interval representations of S-implications.

The paper is organized as follows. In Section II, we discuss the conditions to obtain the best interval representation of a real function and present the related definitions and results. Based on these considerations, we focus attention on the interval extensions of fuzzy t-conorm and fuzzy negation in Sections III and IV, respectively. Further analysis of the properties met by fuzzy S-implications is done in Section V. Section VI shows that minimal properties of fuzzy implications may be extended from interval fuzzy degrees, in a natural way. In addition, in Section VI-A, a commutative diagram relating fuzzy S-implications with interval fuzzy S-implications is also discussed. The action of an interval automorphism on an interval S-implication is analyzed in Section VII. The canonical construction of an interval automorphism from an automorphism is presented 
in Section VII-A, including its best interval representation (Section VII-B) and the relation between interval implications and automorphism (Section VII-C). In Section VIII, we conclude with the main results of this paper and some final remarks.

\section{INTERVAL REPRESENTATIONS}

Consider the real unit interval $U=[0,1] \subseteq \Re$. Let $\mathbb{U}$ be the set of subintervals of $U$, i.e. $\mathbb{U}=\{[a, b] \mid 0 \leq a \leq b \leq$ $1\}$. The interval set has two projections $l, r: \mathbb{U} \rightarrow U$ defined by $l([a, b])=a$ and $r([a, b])=b$, respectively. For $X \in \mathbb{U}$, $l(X)$ and $r(X)$ are also denoted by $\underline{X}$ and $\bar{X}$, respectively.

Several natural partial orders may be defined on $\mathbb{U}$ [9]. The most used orders in the context of interval mathematics and considered in this work, are as it follows:

1) Product: $X \leq Y$ if and only if $\underline{X} \leq \underline{Y}$ and $\bar{X} \leq \bar{Y}$.

2) Inclusion order: $X \subseteq Y$ if and only if $\underline{X} \geq \underline{Y}$ and $\bar{X} \leq \bar{Y}$

An interval $X \in \mathbb{U}$ is said to be an interval representation of a real number $\alpha$ if $\alpha \in X$. Considering two interval representations $X$ and $Y$ of a real number $\alpha, X$ is said a better representation of $\alpha$ than $Y$ if $X$ is narrower than $Y$, that is, if $X \subseteq Y$. This notion could be easily extended for tuples of $n$ intervals $(\vec{X})=\left(X_{1}, \ldots, X_{n}\right)$.

Definition 2.1: A function $F: \mathbb{U}^{n} \longrightarrow \mathbb{U}$ is an interval representation of a function $f: U^{n} \longrightarrow U$ if, for each $\vec{X} \in \mathbb{U}^{n}$ and $\vec{x} \in \vec{X}, f(\vec{x}) \in F(\vec{X})$ [31].

Based on the previous discussion, an interval function may be seen as a representation of a subset of real numbers. Thus, extending the previous definition, an interval function $F$ : $\mathbb{U}^{n} \longrightarrow \mathbb{U}$ is a better interval representation of the function $f: U^{n} \longrightarrow U$ than $G: \mathbb{U}^{n} \longrightarrow \mathbb{U}$, denoted by $G \sqsubseteq F$, if for each $\vec{X} \in \mathbb{U}^{n}$, the inclusion $F(\vec{X}) \subseteq G(\vec{X})$ holds.

\section{A. Best interval representation}

Definition 2.2: For each real function $f: U^{n} \longrightarrow U$, the interval function $\widehat{f}: \mathbb{U}^{n} \longrightarrow \mathbb{U}$ defined by

$$
\widehat{f}(\vec{X})=[\inf \{f(\vec{x}): \vec{x} \in \vec{X}\}, \sup \{f(\vec{x}): \vec{x} \in \vec{X}\}]
$$

is called the best interval representation of $f$ [31].

The interval function $\widehat{f}$ is well defined and for any other interval representation $F$ of $f, F \sqsubseteq \widehat{f}$. The interval function $\widehat{f}$ returns a narrower interval than any other interval representation of $f$. Thus, $\widehat{f}$ has the optimality property of interval algorithms mentioned by Hickey et al. [20], when it is seen as an algorithm to compute a real function $f$.

Notice that if $f$ is continuous in the usual sense, then for each $X \in \mathbb{U}^{n}, \widehat{f}(\vec{X})=\{f(\vec{x}): \vec{x} \in \vec{X}\}=f(\vec{X})$. The main result in [31] can be adapted to our context, i.e. for $U^{n}$ instead of $\Re$, as shown in the following:

Theorem 2.1: Let $f: U^{n} \longrightarrow U$ be a function. The following statements are equivalent: (i) $f$ is continuous; (ii) $\widehat{f}$ is Scott continuous; (iii) $\widehat{f}$ is Moore continuous.

Moore and Scott continuities are the two most common continuity notions used in interval mathematics [31]. Another approach based on Coherence Spaces can be found in [12], [11].

\section{INTERVAL T-CONORM}

Considering the interval generalization proposed in [6], an interval triangular conorm ( $t$-conorm for short) may be considered as an interval representation of a t-conorm. This generalization fit with the fuzzy principle, which means that the interval degree of membership may be thought as an approximation of the exact degree.

Notice that a t-conorm is a function $S: U^{2} \rightarrow U$ which is commutative, associative, monotonic and has 0 as neutral element. In the following definition, an extension of the t-conorm notion for $\mathbb{I}$ is considered, following the same approach introduced in [6].

Definition 3.1: A function $\mathbb{S}: \mathbb{U}^{2} \rightarrow \mathbb{U}$ is an interval t-conorm if it is commutative, associative, monotonic with respect to the product and inclusion order and $[0,0]$ is a neutral element.

Proposition 3.1: If $S$ is a t-conorm then $\widehat{S}: \mathbb{U}^{2} \rightarrow \mathbb{U}$ is an interval t-conorm.

Proof: See [7].

A characterization of $\widehat{S}$ can be expressed by:

$$
\widehat{S}(X, Y)=[S(\underline{X}, \underline{Y}), S(\bar{X}, \bar{Y})]
$$

\section{INTERVAL FUZZY NEGATION}

A function $N: U \rightarrow U$ is a fuzzy negation if

- N1: $N(0)=1$ and $N(1)=0$.

- N2: If $x \geq y$ then $N(x) \leq N(y), \forall x, y \in I$.

In addition, fuzzy negations satisfying the involutive property are called strong fuzzy negations [22], [8]:

- N3: $N(N(x))=x, \forall x \in U$.

Definition 4.1: An interval function $\mathbb{N}: \mathbb{U} \longrightarrow \mathbb{U}$ is an interval fuzzy negation if, for any $X, Y$ in $\mathbb{U}$, the following properties hold:

- $\mathbb{N} 1: \mathbb{N}([0,0])=[1,1]$ and $\mathbb{N}([1,1])=[0,0]$.

- $\mathbb{N} 2$ : If $X \geq Y$ then $\mathbb{N}(X) \leq \mathbb{N}(Y)$.

- $\mathbb{N} 3$ : If $X \subseteq Y$ then $\mathbb{N}(X) \supseteq \mathbb{N}(Y)$.

If $\mathbb{N}$ also meets the involutive property, it is a strong interval fuzzy negation:

- $\mathbb{N} 4: \mathbb{N}(\mathbb{N}(X))=X, \forall X \in \mathbb{U}$.

Let $N: U \longrightarrow U$ be a fuzzy negation. A characterization of $\widehat{N}$ is presented in the following:

$$
\widehat{N}(X)=[N(\bar{X}), N(\underline{X})] .
$$

Theorem 4.1: Let $N: U \longrightarrow U$ be a fuzzy negation. Then $\widehat{N}$ is an interval fuzzy negation. In addition, if $N$ is a strong fuzzy negation then $\widehat{N}$ is a strong interval fuzzy negation.

Proof: $\mathbb{N} 1$ : Trivially, $\mathbb{N} 1$ is satisfied.

$\mathbb{N} 2$ : If $X \geq Y$ then $\bar{Y} \leq \bar{X}$ and $\underline{Y} \leq \underline{X}$. Therefore, by N2, $\widehat{N}(X)=[N(\bar{X}), N(\underline{X})] \leq[N(\bar{Y}), N(\underline{Y})]$ and $\widehat{N}(X) \leq$ $\widehat{N}(Y)$.

NN3: If $X \subseteq Y$ then $\bar{X} \leq \bar{Y}$ and $\underline{Y} \leq \underline{X}$. Therefore, by N2, $\widehat{N}(X)=[N(\bar{X}), N(\underline{X})] \subseteq[N(\bar{Y}), N(\underline{Y})]$ and $\widehat{N}(X) \subseteq$ $\widehat{N}(Y)$. 
$\mathbb{N} 4: \widehat{N}(\widehat{N}(X))=\widehat{N}([N(\bar{X}), N(\underline{X})])$ when $\mathbb{N}$ is a strong negation. Therefore, $\widehat{N}(\widehat{N}(X))=[N(N(\underline{X})), N(N(\bar{X}))]$ and $\widehat{N}(\widehat{N}(X))=X$.

\section{FUZZY IMPLICATION}

Several definitions for fuzzy implication together with related properties have been given (see [2], [4], [8], [15], [17], [21], [25], [30], [33], [34], [35]). The unique consensus in these definitions is that the fuzzy implication should have the same behavior as the classical implication for the crisp case. Thus, a binary function $I: U^{2} \longrightarrow U$ is a fuzzy implication if $I$ meets the minimal boundary conditions:

$$
I(1,1)=I(0,1)=I(0,0)=1 \text { and } I(1,0)=0 .
$$

Several reasonable properties may be required for fuzzy implications. The properties considered in this paper are listed below:

- I1: If $x \leq z$ then $I(x, y) \geq I(z, y)$;

- I2: If $y \leq z$ then $I(x, y) \leq I(x, z)$;

- I3: $I(1, x)=x$ (left neutrality principle);

- I4: $I(x, I(y, z))=I(y, I(x, z))$ (exchange principle);

- I5: $I(x, y)=I(x, I(x, y))$.

\section{A. S-implications}

Let $S$ be a t-conorm and $N$ be a fuzzy negation. Then a fuzzy implication, called S-implication, is given by the equality

$$
I_{S, N}(x, y)=S(N(x), y) .
$$

An S-implication arises from the notion of disjunction and negation using the corresponding tautology of classical logic. Thus, S-implications are based on the classical logical equivalence: $\alpha \rightarrow \beta \equiv \neg \alpha \vee \beta$.

One can notice that in some texts (like, e.g. in [8], [17], [16]), an S-implication requires strong fuzzy negation. As the approach presented in [22], [2], in this work this condition is not required. The main results relating the S-implication and the properties I1, .., I5 are presented in the following.

Proposition 5.1: Let $I: U^{2} \rightarrow U$ be a fuzzy implication. $I$ is an S-implication if and only if the properties I1, I2, I3 and $\mathrm{I} 4$ are met.

Proof: See [3].

Proposition 5.2: An S-implication $I$ generated by a tconorm $S$ and a continuous fuzzy negation $N$ satisfies the property 15 if and only if $S=S_{M}$, where $S_{M}(x, y)=$ $\max (x, y)$ (maximum).

Proof: See corollary 2, in [32].

\section{INTERVAL FUZZY IMPLICATION}

According to the idea that values in interval mathematics are identified with degenerate intervals, the minimal properties of fuzzy implications can be naturally extended from interval fuzzy degrees, when the respective degenerate intervals are considered. Thus, a function $\mathbb{I}: \mathbb{U}^{2} \longrightarrow \mathbb{U}$ is a interval fuzzy implication if the following conditions hold:

- $\mathbb{I}([1,1],[1,1])=\mathbb{I}([0,0],[0,0])=\mathbb{I}([0,0],[1,1])=$ $[1,1]$

- $\mathbb{I}([1,1],[0,0])=[0,0]$.

Some extra properties can be naturally extended.

- $\mathbb{I} 1$ : If $X \leq Z$ then $\mathbb{I}(X, Y) \geq \mathbb{I}(Z, Y)$,

- $\mathbb{I} 2$ : If $Y \leq Z$ then $\mathbb{I}(X, Y) \leq \mathbb{I}(X, Z)$,

- $\mathbb{I} 3: \mathbb{I}([1,1], X)=X$,

- $\mathbb{I} 4: \mathbb{I}(X, \mathbb{I}(Y, Z))=\mathbb{I}(Y, \mathbb{I}(X, Z))$,

- I5a: $\mathbb{I}(X, Y) \subseteq \mathbb{I}(X, \mathbb{I}(X, Y))$

- $\mathbb{I} 5 \mathrm{~b}: \mathbb{I}([x, x], Y)=\mathbb{I}([x, x], \mathbb{I}([x, x], Y))$

Considering any fuzzy implication, it is always possible to obtain canonically an interval fuzzy implication. The interval fuzzy implication also meets the optimality property and preserves the same properties satisfied by the fuzzy implication. In the following two propositions, the best interval representation of a fuzzy implication is shown as an inclusion-monotonic function in both arguments. The related proofs are straightforward, following from the definition of $\widehat{I}$ as a particular case of the equation (1).

Proposition 6.1: If $I$ is a fuzzy implication then $\widehat{I}$ is an interval fuzzy implication.

Proof: See [7].

Proposition 6.2: Let $I$ be a fuzzy implication. Then for each $X_{1}, X_{2}, Y_{1}, Y_{2} \in \mathbb{U}$, if $X_{1} \subseteq X_{2}$ and $Y_{1} \subseteq Y_{2}$ then $\widehat{I}\left(X_{1}, Y_{1}\right) \subseteq \widehat{I}\left(X_{2}, Y_{2}\right)$.

Proof: It is straightforward.

Theorem 6.1: Let $I$ be a fuzzy implication. If $I$ satisfies a property $\mathrm{Ik}$, for some $k=1, \ldots, 5$, then $\widehat{I}$ satisfies the property $\mathbb{I k}$.

Proof: I1: If $u \in \widehat{I}(X, Y)$ then there exists $x \in X$ and $y \in Y$ such as $I(x, y)=u$. If $X \leq Z$ then there exists $z \in Z$ and $x \leq z$. So, by I1, $u=I(x, y) \geq I(z, y)$. On the other hand, if $v \in \widehat{I}(Z, Y)$ then there exists $z \in Z$ and $y \in Y$ such as $I(z, y)=v$. If $X \leq Z$ then $x \leq z$ for some $x \in X$. So, by I1, $I(x, y) \geq I(z, y)=v$. Therefore, for each $u \in \widehat{I}(X, Y)$ there is $v \in \widehat{I}(Z, Y)$ and $u \geq v$. In addition, for each $v \in \widehat{I}(Z, Y)$ there is $u \in \widehat{I}(X, Y)$ such as $u \geq v$. Hence, $\widehat{I}(X, Y) \geq \widehat{I}(Z, Y)$.

I2: If $u \in \widehat{I}(X, Y)$ then there exists $x \in X$ and $y \in Y$ such as $I(x, y)=u$. If $Y \leq Z$ then there exists $z \in Z$ such as $y \leq z$. So, by $\mathrm{I} 2, u=I(x, y) \leq I(x, z)$. On the other hand, if $v \in \widehat{I}(X, Z)$ then there exists $z \in Z$ and $x \in X$ such as $I(x, z)=v$. If $Y \leq Z$ then $y \leq z$ for some $y \in Y$. So, by I2, $I(x, y) \geq I(x, z)=v$. Therefore, for each $u \in \widehat{I}(X, Y)$ there is $v \in \widehat{I}(X, Z)$ such as $u \leq v$ and for each $v \in \widehat{I}(X, Z)$ there is $u \in \widehat{I}(X, Y)$ such as $u \leq v$. Hence, $\widehat{I}(X, Y) \leq \widehat{I}(X, Z)$.

I3: Trivially, by $\mathrm{I} 3$, for each $x \in X, I(1, x)=x$ and so $\{I(1, x): x \in X\}=X$. Thus, since $\widehat{I}([1,1], X)$ is the narrowest interval containing $\{I(1, x): x \in X\}$, then $\widehat{I}([1,1], X)=X$. 
II4: If $u \in \widehat{I}(X, \widehat{I}(Y, Z))$ then there exists $x \in X$, $y \in Y$ and $z \in Z$ such as $I(x, I(y, z))=u$. But, by $\mathrm{I} 4, u=I(y, I(x, z))$. So, $u \in \widehat{I}(Y, \widehat{I}(X, Z))$ and therefore $\widehat{I}(X, \widehat{I}(Y, Z)) \subseteq \widehat{I}(Y, \widehat{I}(X, Z))$. Analogously, if $u \in \widehat{I}(Y, \widehat{I}(X, Z))$ then there exists $x \in X, y \in Y$ and $z \in Z$ such as $I(y, I(y x, z))=u$. But, by I4, $u=I(x, I(y, z))$. So, $u \in \widehat{I}(X, \widehat{I}(X Y, Z))$ and therefore $\widehat{I}(Y, \widehat{I}(X, Z)) \subseteq$ $\widehat{I}(X, \widehat{I}(Y, Z))$. Hence, $\widehat{I}(X, \widehat{I}(Y, Z))=\widehat{I}(Y, \widehat{I}(X, Z))$.

I5 $a$ : If $u \in \widehat{I}(X, Y)$ then there exists $x \in X$ and $y \in Y$ such as $I(x, y)=u$. So, by I5, $u=I(x, I(x, y))$ and therefore $u \in \widehat{I}(X, \widehat{I}(X, Y))$. Hence, $\widehat{I}(X, Y) \subseteq \widehat{I}(X, \widehat{I}(X, Y))$.

$\mathbb{I} 5 b: \quad$ By $\mathbb{I} 10 \mathrm{a}, \widehat{I}([x, x], Y) \subseteq \widehat{I}([x, x], \widehat{I}([x, x], Y))$. So, it only remains to prove that $\widehat{I}([x, x], Y) \supseteq$ $\widehat{I}([x, x], \widehat{I}([x, x], Y))$. Let $u \in \widehat{I}([x, x], \widehat{I}([x, x], Y))$, then there exists $y \in Y$ such as $u=I(x, I(x, y))$. But, by $\mathrm{I} 5, I(x, I(x, y))=I(x, y)$. So, $u \in \widehat{I}([x, x], Y)$ and therefore $\widehat{I}([x, x], Y) \supseteq \widehat{I}([x, x], \widehat{I}([x, x], Y))$.

Proposition 6.3: Let $I: U^{2} \longrightarrow U$ be a fuzzy implication satisfying the properties I1 and I2. Then an characterization of $\widehat{I}$ can be obtained as

$$
\begin{aligned}
\widehat{I}(X, Y)= & {[I(\max (\bar{X}, \bar{Y}), \min (\underline{X}, \underline{Y})),} \\
& I(\min (\underline{X}, \underline{Y}), \max (\bar{X}, \bar{Y}))] .
\end{aligned}
$$

Proof: If $\underline{X} \leq x \leq \bar{X}$ and $\underline{Y} \leq y \leq \bar{Y}$ then $\min (\underline{X}, \underline{Y}) \leq x, y \leq \max (\bar{X}, \bar{Y})$. By the properties I1 and I2, $I(\max (\bar{X}, \bar{Y}), \min (\underline{X}, \underline{Y})) \leq I(x, y) \leq$ $I(\min (\underline{X}, \underline{Y}), \max (\bar{X}, \bar{Y}))$. So, $\mathbb{I}=\{I(x, y) \mid x \in X, y \in$ $Y\} \subseteq \widehat{I}(X, Y)$ and $I(\max (\bar{X}, \bar{Y}), \min (\underline{X}, \underline{Y}))$ are lower and upper bound of $\mathbb{I}$, respectively. Hence, because $I(\min (\underline{X}, \underline{Y}), \max (\bar{X}, \bar{Y}))$ belongs to $\mathbb{I}$, they are the infimum and supremum of $\mathbb{I}$.

\section{A. Interval S-implication}

An interval fuzzy implication $\mathbb{I}_{\mathbb{S}, \mathbb{N}}$ is an interval Simplication if there is an interval t-conorm $\mathbb{S}$ and an interval fuzzy negation $\mathbb{N}$ such as

$$
\mathbb{I}_{\mathbb{S}, \mathbb{N}}(X, Y)=\mathbb{S}(\mathbb{N}(X), Y) .
$$

Theorem 6.2: Let $S$ be a t-conorm and $N$ be a fuzzy negation. Then $\mathbb{I}_{\widehat{S}, \widehat{N}}=\widehat{I_{S, N}}$.

Proof: Let $X, Y \in \mathbb{U}$. Then

$$
\begin{aligned}
\mathbb{I}_{\widehat{S}, \widehat{N}}(X, Y) & =\widehat{S}(\widehat{N}(X), Y)=\widehat{S}([N(\bar{X}), N(\underline{X})], Y) \\
& =[S(N(\bar{X}), \underline{Y}), S(N(\underline{X}), \bar{Y})] \\
& =\left[I_{S, N}(\bar{X}, \underline{Y}), I_{S, N}(\underline{X}, \bar{Y})\right] \\
& =\bar{I}_{S, N}(X, Y) .
\end{aligned}
$$

The next corollary follows directly.

Corollary 6.1: If $I$ is an S-implication then $\widehat{I}$ is an interval S-implication.

The above results together with Theorem 6.2 state the commutativity of the diagram in Figure 1, where $\mathcal{C}(S)$ $(\mathcal{C}(\mathbb{S}))$ denotes the class of (interval) t-conorms, $\mathcal{C}(N)(\mathcal{C}(\mathbb{N})$ ) indicates the class of (interval) fuzzy negation and $\mathcal{C}(I)$ $(\mathcal{C}(\mathbb{I}))$ is the class of (interval) S-implications.

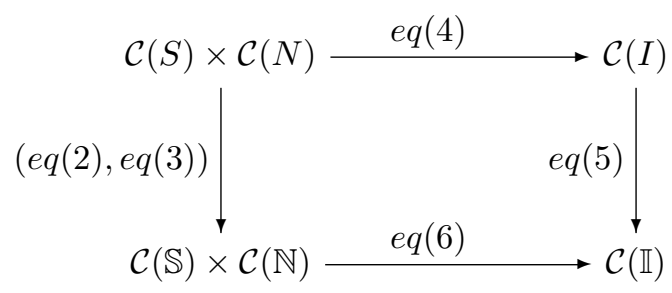

Fig. 1. Commutative diagram relating S-implication with interval Simplications

Proposition 6.4: Let $\mathbb{I}$ be an interval fuzzy implication. $\mathbb{I}$ is an interval $\mathrm{S}$-implication if and only if the properties $\mathbb{I} 1$, $\mathbb{I} 2, \mathbb{I} 3$ and $\mathbb{I} 4$ hold.

Proof: It is analogous to Proposition 5.1.

Proposition 6.5: An interval S-implication $\mathbb{I}$ generated by an interval t-conorm $\mathbb{S}$ and an interval continuous fuzzy negation $\mathbb{N}$ satisfies the property $\mathbb{I} 5 a$ and $\mathbb{I} 5 b$ if and only if $\mathbb{S}=\mathbb{S}_{M}$, where $\mathbb{S}_{M}(X, Y)=\left[S_{M}(\underline{X}, \underline{Y}), S_{M}(\bar{X}, \bar{Y})\right]$.

Proof: It is similar to Proposition 5.2.

\section{INTERVAL AUTOMORPHISM}

Definition 7.1: A mapping $\rho: U \longrightarrow U$ is an automorphism if it is bijective and monotonic ( $x \leq y$ implies that $\rho(x) \leq \rho(y))$ [23], [29]. Aut $(U)$ denotes the set of automorphisms.

An equivalent definition is given in [8], where $\rho: U \longrightarrow U$ is an automorphism if it is a continuous and is a strictly increasing function such that $\rho(0)=0$ and $\rho(1)=1$.

Automorphisms are closed under composition, i.e., if $\rho$ and $\rho^{\prime}$ are automorphisms then $\rho \circ \rho^{\prime}(x)=\rho\left(\rho^{\prime}(x)\right)$ is also an automorphism. In addition, the inverse of an automorphism is also an automorphism.

Let $\rho$ be an automorphism and $I$ be a fuzzy implication. The action of $\rho$ on $I$, denoted by $I^{\rho}$, defined as

$$
I^{\rho}(x, y)=\rho^{-1}(I(\rho(x), \rho(y))),
$$

is a fuzzy implication. Moreover, if $I$ is an S-implication then $I^{\rho}$ is also an S-implication.

\section{A. Canonical construction of an interval automorphism}

A mapping $\varrho: \mathbb{U} \longrightarrow \mathbb{U}$ is an interval automorphism if it is bijective and monotonic w.r.t. the product order [18], [19] ( $X \leq Y$ implies that $\varrho(X) \leq \varrho(Y)$ ). The set of all interval automorphisms $\varrho: \mathbb{U} \longrightarrow \mathbb{U}$ is denoted by $\operatorname{Aut}(\mathbb{U})$.

Theorem 7.1: Let $\varrho: \mathbb{U} \longrightarrow \mathbb{U}$ be an interval automorphism. Then there exists an automorphism $\rho: U \longrightarrow U$ such that

Proof: See Theorem 2 of [18].

$$
\varrho(X)=[\rho(\underline{X}), \rho(\bar{X})] .
$$

The equation (8) also provides a canonical construction of interval automorphisms from automorphisms and therefore a bijection between the sets $A u t(U)$ and $A u t(\mathbb{U})$ (Theorem 3 of [18]). 


\section{B. The best interval representation of an automorphism}

In the following, we will see interval automorphisms from a representation of automorphism point of view.

Theorem 7.2 (Automorphism representation theorem): Let $\rho: U \rightarrow U$ be an automorphism. Then $\hat{\rho}$ is an interval automorphism and its characterization can be obtained as:

Proof: See [6].

$$
\widehat{\rho}(X)=[\rho(\underline{X}), \rho(\bar{X})] .
$$

So, interval automorphisms are the best interval representations of automorphisms.

Notice that t-conorms were required, by definition, to satisfy $\subseteq$-monotonicity. Nevertheless, this property was not required by the definition of interval automorphism. In the following, we show that interval automorphisms also are $\subseteq$ monotonic [6].

Corollary 7.1: If $\varrho$ is an interval automorphism then $\varrho$ is inclusion monotonic, i.e., if $X \subseteq Y$ then $\varrho(X) \subseteq \varrho(Y)$.

Proof: See [6].

Analogously, considering the alternative definition of automorphism used by [8], we can provide alternative characterizations for interval automorphisms based on the Moore and Scott continuity.

Consider $\varrho: \mathbb{U} \longrightarrow \mathbb{U} . \varrho$ is strictly increasing if, for each $X, Y \in \mathbb{U}$, whenever $X<Y$ (i.e., $X \leq Y$ and $X \neq Y$ ) then $\varrho(X)<\varrho(Y)$.

Proposition 7.1: Consider $\varrho: \mathbb{U} \longrightarrow \mathbb{U} . \varrho$ is an interval automorphism iff $\varrho$ is Moore-continuous, strictly increasing, $\varrho([0,0])=[0,0]$ and $\varrho([1,1])=[1,1]$.

Proof: See [6].

Corollary 7.2: Let $\varrho: \mathbb{U} \longrightarrow \mathbb{U}$ be a Moore-continuous and strictly increasing function such that $\varrho([0,0])=[0,0]$ and $\varrho([1,1])=[1,1]$. Then there exists an automorphism $\rho$ such that $\varrho=\widehat{\rho}$.

Proof: See [6].

The case of Scott-continuity follows the same setting.

Lemma 7.1: Let $\varrho_{1}$ and $\varrho_{2}$ be interval automorphisms. Then $\left(\varrho_{1} \circ \varrho_{2}\right)^{-1}=\varrho_{2}^{-1} \circ \varrho_{1}^{-1}$.

Proof: See [6].

\section{Interval automorphism acting on interval S-implication}

In the following theorem, we will show how interval automorphisms act on interval S-implications, generating new interval S-implications.

Theorem 7.3: Let $\varrho: \mathbb{U} \longrightarrow \mathbb{U}$ be an interval automorphism and $\mathbb{I}: \mathbb{U}^{2} \longrightarrow \mathbb{U}$ be an interval S-implication. Then the mapping $\mathbb{I}^{\varrho}: \mathbb{U}^{2} \longrightarrow \mathbb{U}$ defined by

$$
\mathbb{I}^{\varrho}(X, Y)=\varrho^{-1}(\mathbb{I}(\varrho(X), \varrho(Y)))
$$

is an interval S-implication.

Proof:

$$
\begin{aligned}
\mathbb{I}^{\varrho}(X, Y) & =\varrho^{-1}(\mathbb{I}(\varrho(X), \varrho(Y))) \text { Equation }(10) \\
& =\varrho^{-1}(\mathbb{S}(\mathbb{N}(\varrho(X), \varrho(Y)))) \text { Equation }(6) \\
& =\varrho^{-1}\left(\mathbb{S}\left(\varrho \circ \varrho{ }^{-1}\right)(\mathbb{N}(\varrho(X), \varrho(Y)))\right) \\
& =\varrho^{-1}\left(\mathbb{S}\left(\varrho\left(\mathbb{N}^{\varrho}(X), \varrho(Y)\right)\right)\right) \\
& \left.=\mathbb{S}^{\varrho}\left(\varrho\left(\mathbb{N}^{\varrho}(X), Y\right)\right)\right)
\end{aligned}
$$

Proposition 7.2: Let $\mathbb{I}$ be an interval S-implication and $\varrho_{1}$ and $\varrho_{2}$ be interval automorphisms. Then

$$
\left(\mathbb{I} \varrho_{1}\right) \varrho_{2}=\mathbb{I} \varrho_{1} \circ \varrho_{2} .
$$

$$
\begin{aligned}
& \text { Proof: } \\
& \left(\mathbb{T}^{\varrho_{1}}\right)^{\varrho_{2}}(X, Y)=\varrho_{2}^{-1}\left(\mathbb{T}^{\varrho_{1}}\left(\varrho_{2}(X), \varrho_{2}(Y)\right)\right) \\
& =\varrho_{2}^{-1}\left(\varrho_{1}^{-1}\left(\mathbb{T}\left(\varrho_{1}\left(\varrho_{2}(X)\right), \varrho_{1}\left(\varrho_{2}(Y)\right)\right)\right)\right) \\
& =\varrho_{2}^{-1} \circ \varrho_{1}^{-1}\left(\mathbb{T}\left(\varrho_{1} \circ \varrho_{2}(X), \varrho_{1} \circ \varrho_{2}(Y)\right)\right) \\
& =\left(\varrho_{1} \circ \varrho_{2}\right)^{-1}\left(\mathbb{T}\left(\varrho_{1} \circ \varrho_{2}(X), \varrho_{1} \circ \varrho_{2}(Y)\right)\right) \\
& \text { Lemma } 7.1 \\
& =\mathbb{T}^{\varrho_{1} \varrho_{2}}(X, Y)
\end{aligned}
$$

Theorem 7.4: Let $I$ be a an S-implication and $\rho$ be an automorphism. Then

$$
\widehat{I^{\rho}}=\widehat{I}^{\widehat{\rho}}
$$

Proof:

$$
\begin{aligned}
& \widehat{I}^{\rho}(X, Y)=\left[I^{\rho}(\max (\bar{X}, \bar{Y}), \min (\underline{X}, \underline{\bar{Y}}))\right. \text {, } \\
& \left.I^{\rho}(\min (\underline{X}, \underline{Y}), \max (\bar{X}, \bar{Y}))\right] \\
& \text { Equation }(5) \\
& =\left[\rho^{-1}(I(\rho(\max (\bar{X}, \bar{Y})), \rho(\min (\underline{X}, \underline{Y}))))\right. \text {, } \\
& \left.\rho^{-1}(I(\rho(\min (\underline{X}, \underline{Y})), \rho(\max (\bar{X}, \bar{Y}))))\right] \\
& \text { Equation }(7) \\
& =\widehat{\rho^{-1}}[I(\max (\rho(\bar{X}), \rho(\bar{Y})), \min (\rho(\underline{X}), \rho(\underline{Y}))) \\
& I(\min (\rho(\underline{X}), \rho(\underline{Y})), \max (\rho(\bar{X}), \rho(\bar{Y})))] \\
& \text { Definition }(7.1) \\
& =\widehat{\rho}^{-1}[I(\max (\overline{\widehat{\rho}(X)}, \overline{\hat{\rho}(Y)}), \min (\widehat{\rho}(X), \widehat{\rho}(Y))) \text {, } \\
& I(\min (\widehat{\rho}(X), \hat{\rho}(Y)), \max (\overline{\hat{\rho}(X)}, \overline{\hat{\rho}(Y)})] \\
& \operatorname{Remark} \overline{(5.1)[7] \text { and Equation(9) }} \\
& \left.=\widehat{\rho}^{-1}(\widehat{I}(\widehat{\rho}(X), \widehat{\rho}(Y)))\right) \text { Equation(7) } \\
& =\widehat{I}^{\widehat{\rho}}(X, Y)
\end{aligned}
$$

According to Theorem 7.4, the commutative diagram pictured in Figure 2 holds.

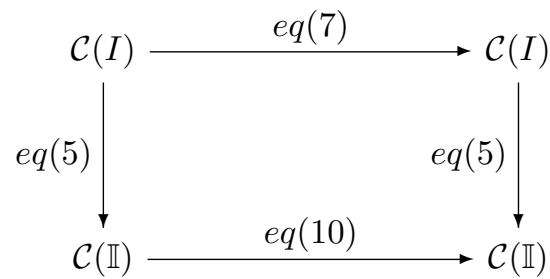

Fig. 2. Commutative diagram relating S-implication, automorphisms, interval S-implications and interval automorphisms

Based on Theorem 7.4, (interval) S-implications and (interval) automorphisms can be seem as objects and morphism, respectively, of the category $\mathfrak{C}(\mathcal{C}(I)$, Aut $(I))$ $(\mathfrak{C}(\mathcal{C}(\mathbb{I}), A u t(\mathbb{I})))$, respectively. In a categorical approach, the action of interval automorphism on interval $\mathrm{S}$ implication can be conceived as a covariant functor whose application over the $\mathrm{S}$-implications and automorphisms in $\mathfrak{C}(\mathcal{C}(I), A u t(I))$ returns the related best interval representations in $\mathfrak{C}(\mathcal{C}(\mathbb{I}), \operatorname{Aut}(\mathbb{I}))$.

\section{FINAL REMARKS}

This work emphasized that both interval mathematics and fuzzy set theory are firmly integrated with principles 
of information theory used to underlying logic system for expert systems. Thus, this paper complements the results of previous works [6] in order to extend the generalization of the main properties of interval fuzzy S-implication and interval automorphisms. The interval extension considers the best interval representation of a real function to deal with the imprecision of a specialist in providing an exact value to measure membership uncertainty.

Throughout this paper, intervals were used to model the uncertainty of a specialist's information related to truth values in the fuzzy propositional calculus: the basic systems are based on interval t-conorm, i.e., using subsets of the real unit interval as the standard sets of truth degrees and applying continuous t-conorms and negation as standard truth interval functions, the standard truth interval function of an S-implication can be obtained.

In addition, we mainly discussed under which conditions generalized fuzzy S-implications applied to interval values preserve properties of canonical forms generated by interval t-conorms. It was shown that properties of fuzzy logic may be naturally extended for interval fuzzy degrees considering the respective degenerate intervals. The significance of interval fuzzy S-implication was emphasized, showing that Simplications can be constructed from interval automorphisms that are preserved by the interval canonical representation.

These results are important not only to analyze deductive systems in mathematical depth but also as foundations of methods based on interval fuzzy logic. They integrate two important features: the accuracy criteria and the optimality property of interval computations, and a formal mathematical theory for the representation of uncertainty, concerned with fuzzy set theory. The former gives a more reliable modelling of real systems and the latter is crucial for their management and control.

\section{ACKNOWLEDGMENTS}

This work was supported in part by the Brazilian funding agencies CNPq (under the process numbers 470871/2004-0 and 470556/2004-8 of Edital Universal) and FAPERGS.

\section{REFERENCES}

[1] G. Alefeld and J. Herzberger, Introduction to Interval Computations, NY: Academic Press, 1983.

[2] M. Baczynski, "Residual Implications Revisited, Notes on the SmetsMagrez," Fuzzy Sets and Systems, vol. 145, no. 2, pp. 267-277, 2004.

[3] M. Baczynski and B. Jayaran, "On the characterization of $(\mathrm{S}, \mathrm{N})$ implications generated from continuous negations," Proc. 11th Conf. on Information Processing and Management of Uncertainty in Knowledgebased Systems, Paris, July 2003, pp. 523-528.

[4] J. Balasubramaniam, "Yager's New Class of Implications $J_{f}$ and Some Classical Tautologies," Information Sciences, 2006.

[5] L.V. Barboza, G.P. Dimuro, and R. H. S. Reiser, "Power Flow with Load Uncertainty," Proc. 8th IEEE Intl. Conf. on Probability Methods Applied to Power Systems, Ames, Sept. 2004.

[6] B.R.C. Bedregal and A. Takahashi, "The Best Interval Representation of T-Norms and Automorphisms," Fuzzy Sets and Systems, vol. 157, no. 24, pp. 3220-3230, 2006.

[7] B.R.C. Bedregal and A. Takahashi, "Interval Valued Versions of TConorms, Fuzzy Negations and Fuzzy Implications," Proc. IEEE Intl. Conf. on Fuzzy Systems, Vancouver, 2006, pp. 1981-1987.
[8] H. Bustince, P. Burilo, and F. Soria, "Automorphism, Negations and Implication Operators," Fuzzy Sets and Systems, vol. 134, pp. 209-229, 2003.

[9] R. Callejas-Bedregal and B.R.C. Bedregal, "Intervals as a Domain Constructor", TEMA - Tendências em Matemática Aplicada e Computacional, vol. 2, no. 1, pp. 43-52, 2001.

[10] G. Cornelis, G. Deschrijver, and E.E. Kerre, "Advances and Challenges in Interval-Valued Fuzzy Logic," Fuzzy Sets and Systems, vol. 157, pp. 622-627, 2006

[11] G.P. Dimuro, A.C.R. Costa, and D.M. Claudio, "A Bi-Structured Coherence Space for a Global Representation of the System IR of Real Intervals," Proc. Intl. Conf. on Information Technology, Bhubaneswar, 1999.

[12] G.P. Dimuro, A.C.R. Costa, and D.M. Claudio, "A Coherence Space of Rational Intervals for a Construction of IR," Reliable Computing, vol. 6, no. 2, pp. 139-178, 2000.

[13] G.P. Dimuro and A.C.R. Costa, "Interval-based Markov Decision Processes for Regulating Interactions Between Two Agents in Multiagent Systems," Springer Lecture Notes in Computer Science, n. 3732 , pp. 102-111, 2005 (Proc. Work. of the State-of-the-Art in Scientific Computing, Lyngby, 2004).

[14] D. Dubois and H. Prade, "Random sets and fuzzy interval analysis," Fuzzy Sets and Systems, vol. 12, pp. 87-101, 1991.

[15] J.C. Fodor, "On Fuzzy Implication Operators," Fuzzy Sets and Systems, vol. 42, pp. 293-300, 1991.

[16] J.C. Fodor, "Contrapositive Symmetry of Fuzzy Implications," Fuzzy Sets and Systems, vol. 69, pp. 141-156, 1995.

[17] J.C. Fodor and M. Roubens, Fuzzy Preference Modelling and Multicriteria Decision Support, Dordrecht: Kluwer Academic Publisher, 1994.

[18] M. Gehrke, C. Walker, and E. Walker, "Some comments on interval valued fuzzy sets," International Journal of Intelligent Systems, vol. 11, pp. 751-759, 1996.

[19] M. Gehrke, C. Walker, and E. Walker, "Algebraic aspects of fuzzy sets and fuzzy logics," Proc. Work. on Current Trends and Development in Fuzzy Logic, Thessaloniki-Greece, Oct. 1998, pp. 101-170.

[20] T. Hickey, Q. Ju, and M. Emdem, "Interval arithmetic: From principles to implementation," Journal of the ACM, vol. 48, no.5, pp. 1038-1068, 2001.

[21] R. Horcik and M. Navara, "Validation Sets in Fuzzy Logics," Kybernetika, vol.38, no.2, pp.319-326, 2002.

[22] E.P. Klement, R. Mesiar, and E. Pap, Triangular Norms, Dordrecht: Kluwer Academic Publisher, 2000.

[23] E. Klement and M. Navara, "A survey on different traingular normbased fuzzy logics," Fuzzy Sets and Systems, vol. 101, pp. 241-251, 1999.

[24] V. Kreinovich and M. Mukaidono, "Interval (pairs of fuzzy values), triples, etc.: Can we thus get an arbitrary ordering? ", Proc. 9th IEEE Intl. Conf. on Fuzzy Systems, San Antonio, TX, 2000, pp. 234-238.

[25] J.M. Leski, " $\varepsilon$-Insensitive Learning Techniques for Approximate Reasoning System," International Journal of Computational Cognition, vol. 1, no. 1, pp. 21-77, 2003.

[26] W. Lodwick, "Preface," Reliable Computing, vol. 10, no. 4, pp. 247248, 2004.

[27] R. Moore, Methods and Applications of Interval Analysis, Philadelphia: SIAM Press, 1979.

[28] R. Moore and W.A. Lodwick, "Interval analysis and fuzzy set theory, "Fuzzy Sets and Systems, vol. 135, no. 1, pp. 5-9, 2003.

[29] M. Navara, "Characterization of measures based on strict triangular norms," J. Math. Anal. Appl., vol. 236, pp. 370-383, 1999.

[30] D. Ruan and E.E. Kerre, "Fuzzy implication operators and generalized fuzzy methods of cases," Fuzzy Sets and Systems, vol. 54, pp. 23-37, 1993.

[31] R.H.N. Santiago, B.R.C. Bedregal, and B.M. Acióly, "Formal Aspects of Correctness and Optimality in Interval Computations," Formal Aspects of Computing, vol. 18, no. 2, pp. 231-243, 2006.

[32] Y. Shi, D. Ruan, and E.E. Kerre,"On the characterizations of fuzzy implications satisfying $I(x, y)=I(x, I(x, y))$ ", Information Sciences, 2007. (in press, doi:10.1016/j.ins.2007.01.026)

[33] R.R. Yager, "On the implication operator in fuzzy logic," Information Sciences, vol.31, pp. 141-164, 1983

[34] R.R. Yager, "On global requirements for implication operators in fuzzy modus ponens," Fuzzy Sets and Systems, vol. 106, pp. 3-10, 1999.

[35] R.R. Yager, "On some new classes of implication operators and their role in approximate reasoning," Information Sciences, vol. 167, pp. 193 216, 2004. 